\title{
Aechmea novoae (Bromeliaceae, Bromelioideae), a Novelty from the State of Jalisco, Mexico
}

\author{
Alejandra Flores-Argüelles, ${ }^{1,2}$ Ana Rosa López-Ferrari, ${ }^{1}$ and Adolfo Espejo-Serna ${ }^{1 *}$ \\ ${ }^{1}$ Herbario Metropolitano, Departamento de Biología, División de Ciencias Biológicas y de la Salud. \\ Universidad Autónoma Metropolitana, Unidad Iztapalapa, Apartado Postal 55-535, C.P. 09340, \\ Ciudad de México, Mexico.
}

${ }^{2}$ Maestría en Biología, Departamentos de Biología e Hidrobiología, División de Ciencias Biológicas y de la Salud. Universidad Autónoma Metropolitana, Unidad Iztapalapa, Apartado Postal 55-535,

C.P. 09340, Ciudad de México, Mexico.

*Author for correspondence: aes@xanum.uam.mx

Abstract. Aechmea novoae Flores-Arg., López-Ferr. \& Espejo, an epiphytic species distributed in the municipalities of Cabo Corrientes, La Huerta, and Puerto Vallarta in the state of Jalisco, Mexico, is described and illustrated. Morphologically, the new taxon resembles $A$. mexicana Baker and $A$. lueddemanniana (K. Koch) Brongn. ex Mez. A comparative table, figures, and a distribution map of the three species are included.

Resumen. Se describe e ilustra Aechmea novoae FloresArg., López-Ferr. \& Espejo, especie epífita que se distribuye en los municipios de Cabo Corrientes, La Huerta y Puerto Vallarta, en el estado de Jalisco, México. El nuevo taxon se parece morfológicamente a A. mexicana Baker y a A. lueddemanniana (K. Koch) Brongn. ex Mez. Se incluye una tabla comparativa, figuras y un mapa de distribución de las tres especies.

Key words: Aechmea, Bromeliaceae, Cabo Corrientes, epiphytes, La Huerta, Mexico.

As a result of botanical explorations in the vicinity of Las Juntas y Los Veranos in the municipality of Cabo Corrientes, Jalisco, Mexico, in the course of the first author's master's thesis project, material in fruit of a species of Aechmea Ruiz \& Pav. (Bromeliaceae, Bromelioideae) was collected. It was originally identified as A. mexicana Baker. However, a detailed examination of herbarium material, as well as the study of a flowering specimen of a cultivated plant from Jalisco, revealed that it is an undescribed species.

\section{Materials and Methods}

Material was collected following the guidelines of Aguirre León (1986); the specimens were deposited at Herbario Metropolitano (UAMIZ), Universidad Autónoma Metropolitana. Around 80 herbarium sheets of Aechmea mexicana and ca. 74 herbarium sheets of $A$. lueddemanniana (K. Koch) Brongn. ex Mez (Appendix 1) from the following herbaria were reviewed: CHIP, CIB, CICY, CORU, ENCB, GH, HEM, IBUG, IEB, K, MEXU, MICH, MO, SERO, UAMIZ, UC, US, and XAL (acronyms according to Thiers, 2020+). Specimens and type images from HAL, K, and UC were also studied. Morphological analyses of the leaves and flowers were made with a standard stereoscope. The description of morphological characters follows Brown and Terry (1992) and Scharf and Gouda (2008).

\section{Taxonomic Treatment}

Aechmea novoae Flores-Arg., López-Ferr. \& Espejo, sp. nov. TYPE: Mexico. Jalisco: municipio de Cabo Corrientes, ejido Las Juntas y Los Veranos, santuario las Guacamayas, $20^{\circ} 25^{\prime} 58.8^{\prime \prime} \mathrm{N}, 105^{\circ} 18^{\prime} 55.8^{\prime \prime} \mathrm{W}, 600$ m.s.l., bosque tropical subcaducifolio, 23 Mar. 2018 (st.), cultivated and pressed 28 Oct. 2019 (fl.), A. Flores-Argüelles, A. Espejo, A. R. López-Ferrari, R. Hernández-Cárdenas \& I. N. Gomez-Escamilla 987 (holotype, UAMIZ!; isotype, IBUG!). Figure 1B-G.

Diagnosis. Aechmea novoae Flores-Arg., López-Ferr. \& Espejo differs from $A$. mexicana Baker by its longer leaf sheaths and blades and cylindrical, sparsely white-lepidote inflorescence (vs. conical, densely white-lepidote), with shorter primary branches and fewer flowers per branch.

Herbs perennial, epiphytic, rarely lithophytic, in flower up to $1.1 \mathrm{~m}$ high from the base of the rosette to the apex of the inflorescence. Rosettes solitary, tank-type, forming an inverted straight cone up to $1.7 \mathrm{~m}$ diam. Stem rhizomatous, appressed to the phorophyte, 5-6 cm diam. and up to $60 \mathrm{~cm}$ long. Roots fibrous, thin. Leaves numerous, 60-160 cm; leaf sheath straw-colored or light brown, elliptic, 21-37 × 13-19.5 cm, entire, densely brownish punctulate lepidote adaxially, sparsely lepidote abaxially; blade green, the lower ones reflexed and 


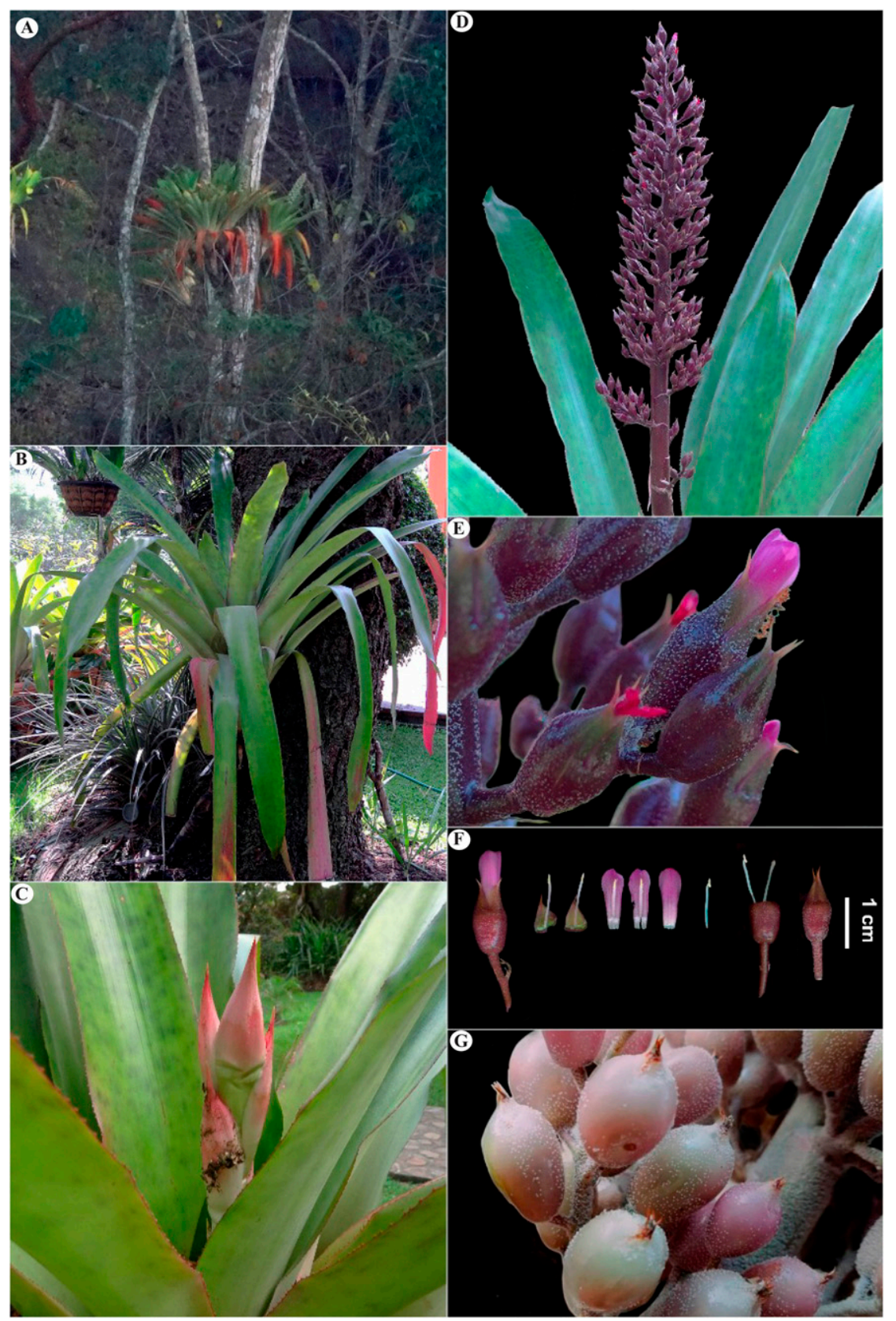

Figure 1. Aechmea novoae Flores-Arg., López-Ferr. \& Espejo. —A. Habit. —B. Plant. —C. Young inflorescence. —D. Mature inflorescence. —E. Inflorescence detail. —F. Dissected flower. —G. Fruits. A based on a photo by A. Espejo-Serna without specimen; B-G based on A. Flores-Argüelles et al. 987 (UAMIZ). Photos: A. Espejo-Serna.

red at the distal portion, strap-shaped, 40-122 $\times$ 9-13.5 cm, coriaceous, sparsely white-lepidote adaxially, densely white-lepidote abaxially, acuminate, margin spinose-serrate, the spines antrorse, 0.8-2.1 $\mathrm{mm}$. Inflorescence terminal, dark purple, erect, paniculiform, 2 or 3 times branched, cylindrical, $70-80 \mathrm{~cm}$, ca. $8 \mathrm{~cm}$ diam., very sparsely white-lepidote; peduncle erect, cylindrical, 35-40 cm, ca. $2 \mathrm{~cm}$ diam.; peduncle bracts red when young, foliaceous, longer than the internodes, the sheaths of the basal ones elliptic, the apical ones narrowly elliptic, 8.1-13 × 1.7-4.4 cm, glabrous, the blades triangular, $1.3-6 \times 0.4-2 \mathrm{~cm}$, 


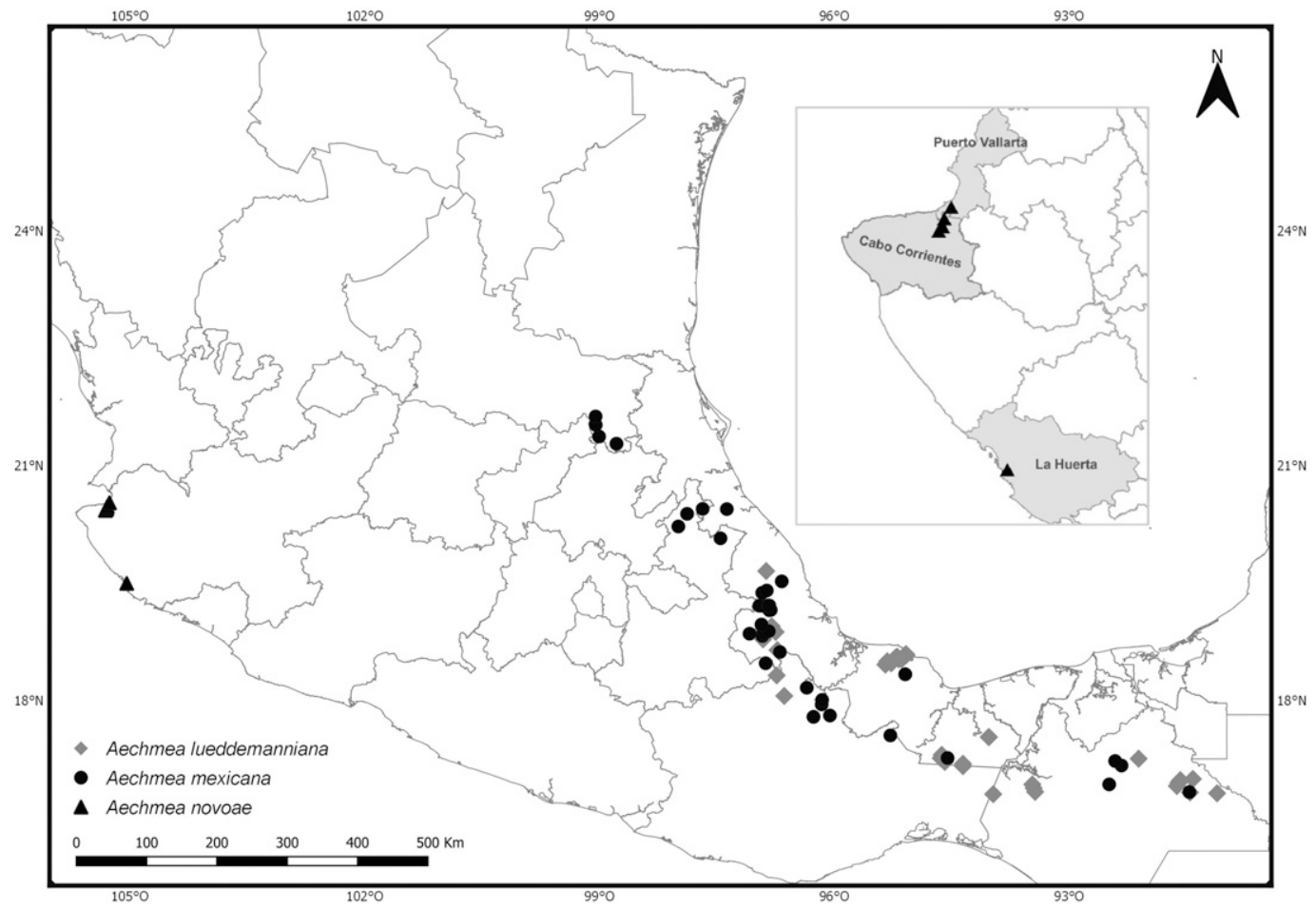

Figure 2. Distribution map of Aechmea. - Aechmea mexicana Baker. — $\Delta$ Aechmea novoae Flores-Arg., López-Ferr. \& Espejo. — Aechmea lueddemanniana (K. Koch) Brongn. ex Mez.

entire, acuminate and pungent at the apex, lepidote on both sides; branches divaricate to ascending, the basal ones $5-6.5 \mathrm{~cm}$ and conspicuously branched, the apical ones 3-4 cm, simple or few-branched; basal primary bracts similar to those of the peduncle, deciduous, the blades ca. $5.8 \times$ ca. $1.6 \mathrm{~cm}$, the apical ones pale brown, filiform, deciduous, $5.5-9 \mathrm{~mm}$; floral bracts similar to equal to the upper primary bracts, $2.2-5 \mathrm{~mm}$; flowers polystichous, ascending to erect, laxly disposed, 2 to 16 per branch, pedicellate; pedicels longer than floral bracts, 2.6-7.7 mm, sparsely white-lepidote. Flowers with sepals connate at the base forming a hypanthium with the ovary, free in the apical portion, asymmetric, with a hyaline wing at the right side, purplish brown, 6.6-10.4 $\times$ 3.5-5.5 mm, fleshy, strongly appressed to the corolla, apiculate, the apicule pale brown, $2-4 \mathrm{~mm}$, persistent in fruit; petals free, lilac to magenta, oblong to oblong-spathulate, $13-16 \times 3-4.5 \mathrm{~mm}$, rounded at the apex, bearing a pair of saclike basal appendages on the adaxial surface formed by 2 lateral folds and 2 fimbriate ligules, $1.5 \mathrm{~mm}$; stamens equal, shorter than petals, filaments white, $2.3-7.8 \mathrm{~mm}, 3$ adnate basally to the petals, anthers yellow, oblong, 1.2-2 $\mathrm{mm}$; ovary oblong to obconic, $8.7-10 \mathrm{~mm}$, ca. $6.2 \mathrm{~mm}$ diam., style filiform, 8-10 mm, stigma simple, erect. Fruit a berry, pale green when young, pale rose to whitish when mature, obovoid, ca. $20 \mathrm{~mm}, 5.8-8.4 \mathrm{~mm}$ diam.; seeds dark brown, fusiform, curved, 2-2.6 mm.

Distribution and habitat. Aechmea novoae is known at this time from the municipalities of Cabo Corrientes, La Huerta, and Puerto Vallarta, Jalisco, Mexico (Fig. 2), growing as epiphyte in tropical subdeciduous forests and gallery forests, at 100-600 m elevation.

Phenology. This species flowers from September to January, fruiting from November to March.

Etymology. The specific epithet honors Jorge Heriberto Novoa-Ramos, a dear friend who has made great efforts for the conservation of santuario Las Guacamayas in Cabo Corrientes Municipality. Jorge guided and helped during the fieldwork of the first author's thesis project.

Notes. Aechmea novoae was for a long time confused with A. mexicana (Magaña R., 1986; McVaugh, 1989; Vázquez-García et al., 2000; Espejo-Serna et al., 2004; Ramírez Delgadillo et al., 2010), a species that it closely resembles morphologically. However, it differs by its longer leaves (sheaths and blades), and by its cylindrical, sparsely white-lepidote inflorescence (Figs. 1, 3; Table 1). Magaña (1986) included A. novoae under the name of $A$. mexicana in her thesis about the Bromeliaceae 


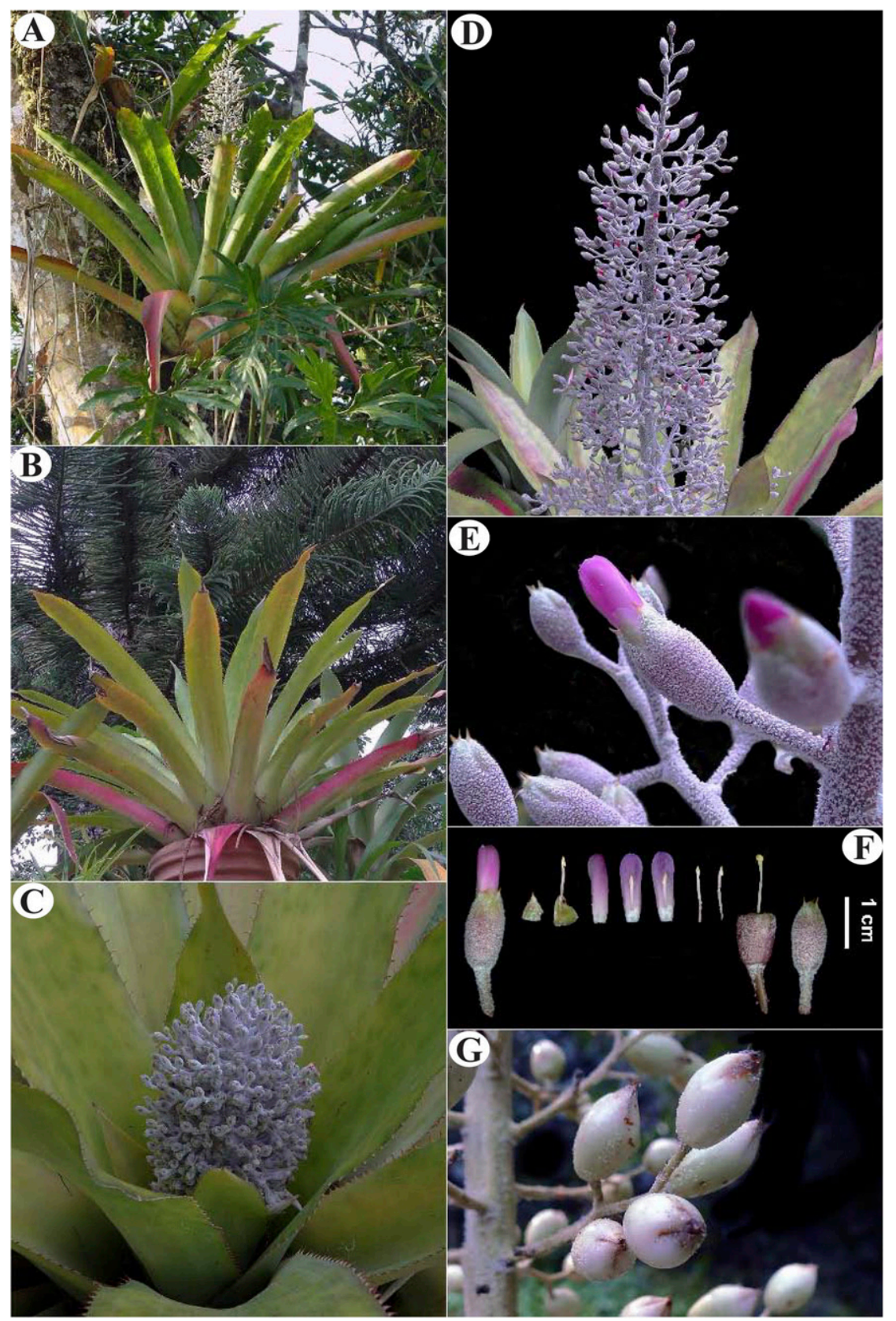

Figure 3. Aechmea mexicana Baker. - A. Habit. —B. Plant. —C. Young inflorescence. -D. Mature inflorescence. —E. Inflorescence detail. —F. Flower dissected. —G. Fruits. Based on M. Miguel-Vázquez et al. 258 (UAMIZ). Photos: A. EspejoSerna.

of the Coast of Jalisco, describing it as a plant with narrow inflorescences, $7 \mathrm{~cm}$ wide (diameter) with basal branches 4-5 cm long. Later, McVaugh (1989), in Flora Novo-Galiciana, refers to Magaña's work, indicating also that plants from Jalisco have inflorescences with basal branches up to $4-5 \mathrm{~cm}$ long, a characteristic that clearly distinguishes these plants from A. mexicana whose basal branches reach $18.5 \mathrm{~cm}$ long. It is important to note that Magaña's description was based only on two fruiting specimens (Magaña \& Lott 45 and Beutelspacher 38662, see paratypes below). 
Table 1. Comparative characteristics of Aechmea novoae Flores-Arg., López-Ferr. \& Espejo, A. mexicana Baker, and A. lueddemanniana (K. Koch) Brongn. ex Mez.

\begin{tabular}{|c|c|c|c|}
\hline & A. novoae & A. mexicana & A. lueddemanniana \\
\hline $\begin{array}{l}\text { Distribution (States of } \\
\text { Mexico) }\end{array}$ & Jalisco & $\begin{array}{l}\text { Chiapas, Oaxaca, Puebla, San } \\
\text { Luis Potosí, and Veracruz }\end{array}$ & $\begin{array}{l}\text { Chiapas, Oaxaca, and } \\
\text { Veracruz }\end{array}$ \\
\hline Foliar sheath size & $21-37 \times 13-19.5 \mathrm{~cm}$ & $14-20 \times 8-16 \mathrm{~cm}$ & $6-10 \times 5-6 \mathrm{~cm}$ \\
\hline Foliar blade size & $40-122 \times 9-13.5 \mathrm{~cm}$ & $54-100 \times 5-16 \mathrm{~cm}$ & $25-55 \times 3.5-6.5 \mathrm{~cm}$ \\
\hline $\begin{array}{l}\text { Inflorescence form and } \\
\text { length }\end{array}$ & cylindrical, $70-80 \mathrm{~cm}$ & conical, 80-90 cm & $\begin{array}{l}\text { conical to cylindrical, } 40-50 \\
\mathrm{~cm}\end{array}$ \\
\hline $\begin{array}{l}\text { Inflorescence color and } \\
\text { indument }\end{array}$ & $\begin{array}{l}\text { dark purple, very sparsely white- } \\
\text { lepidote }\end{array}$ & green, densely white-lepidote & $\begin{array}{l}\text { dark purple, sparsely white- } \\
\text { lepidote }\end{array}$ \\
\hline Peduncle bract color & $\begin{array}{l}\text { reddish when young, brownish } \\
\text { when mature }\end{array}$ & $\begin{array}{l}\text { whitish when young, pale brown } \\
\text { when mature }\end{array}$ & $\begin{array}{l}\text { whitish when young, pale } \\
\text { brown when mature }\end{array}$ \\
\hline $\begin{array}{l}\text { Length of basal branches } \\
\text { of inflorescence }\end{array}$ & $5-6.5 \mathrm{~cm}$ & $15-18.5 \mathrm{~cm}$ & $4-5 \mathrm{~cm}$ \\
\hline Flowers per branch & 2 to 16 & 5 to 56 & 3 to 6 \\
\hline Sepal color and size & $\begin{array}{l}\text { purplish brown, } 6.6-10.4 \times \\
3.5-5.5 \mathrm{~mm}\end{array}$ & green, $5.3-7.3 \times 3.7-5.2 \mathrm{~mm}$ & $\begin{array}{l}\text { purplish brown, } 3-5 \times \\
2-4 \mathrm{~mm}\end{array}$ \\
\hline Petal size & $13-16 \times 3-4.5 \mathrm{~mm}$ & $13-14 \times 5-5.5 \mathrm{~mm}$ & $8-10 \times 3-4 \mathrm{~mm}$ \\
\hline Fruit color & $\begin{array}{l}\text { pale green when young, pale rose } \\
\text { to whitish when mature }\end{array}$ & $\begin{array}{l}\text { green when young, whitish purple } \\
\text { when mature }\end{array}$ & $\begin{array}{l}\text { white when young, purple to } \\
\text { black when mature }\end{array}$ \\
\hline $\begin{array}{l}\text { Fruit shape, size at } \\
\text { maturity }\end{array}$ & obovoid, ca. $20 \times 5.8-8.4 \mathrm{~mm}$ & ellipsoid, $15-17 \times 7-8 \mathrm{~mm}$ & oblong, 11-15 × 3-7 mm \\
\hline
\end{tabular}

Aechmea novoae and A. mexicana belong to the subgenus Podaechmea Mez (1896), which was treated as a genus by Smith and Kress $(1989,1990)$. The third Mexican member of Aechmea subg. Podaechmea is A. lueddemanniana (Espejo-Serna \& López-Ferrari, 1994), a taxon that shares with the other two species compound inflorescences, lepidote-pedicellate flowers, and basal petal appendages (Smith \& Downs, 1979), but differs from both by its much smaller habit (Fig. 4, Table 1). Aechmea novoae also differs from both A. lueddemanniana and $A$. mexicana by its geographic distribution, Jalisco State in western Mexico, whereas the other two species are reported from Chiapas, Oaxaca, Puebla, San Luis Potosí, and Veracruz in the eastern slope of the country (Fig. 2).

Recently, based on molecular data, the Androlepis Alliance was proposed including Aechmea subg. Podaechmea among other taxa with different morphological characters (Sass \& Specht, 2010; Ramírez-Díaz et al., 2019). As a member of Aechmea subg. Podaechmea, the new species here proposed obviously also belongs to the Androlepis Alliance.

With this finding, the number of Mexican Aechmea species increases to 10 , three of them endemic to the country (Espejo-Serna et al., 2004; López-Ferrari et al., 2011; Espejo Serna, 2012).

Paratypes. MEXICO. Jalisco: municipio de Cabo Corrientes, ejido Las Juntas y Los Veranos, santuario las Guacamayas, $20^{\circ} 25^{\prime} 49.3^{\prime \prime} \mathrm{N}, 105^{\circ} 18^{\prime} 59.54^{\prime \prime} \mathrm{W}, 586$ m.s.l., vegetación riparia, 24 Mar. 2019 (fr.), J. Novoa-Ramos s.n. (UAMIZ); $24 \mathrm{~km}$ al S de Puerto Vallarta, sobre la carretera a Barra de Navidad, cañada con selva mediana subcaducifolia, 18 Jan. 1985 (fr.), P. Magaña \& E. Lott 45 (MEXU 414700, 414701); municipio de La Huerta, cerca de estación Chamela, 100 m.s.l., 5 Feb. 1975 (fr.), C. Beutelspacher 38662 (MEXU 182308).

Photographic records. MEXICO. Jalisco: municipio de Cabo Corrientes, Las Juntas y Los Veranos, 20²8'53.61"N, $105^{\circ} 17^{\prime} 37.06^{\prime \prime} \mathrm{W}$, ca. 290 m.s.l., 22 Mar. 2018 (fr.), photo by A. Espejo (UAMIZ) (Fig. 1A); municipio de Puerto Vallarta, camino al Edén, 20³1'37.56"N, $105^{\circ} 15^{\prime} 59.76^{\prime \prime} \mathrm{W}, 130$ m.s.l., Jan. 2019 (fr.), photo by Dante S. Figueroa (UAMIZ).

Acknowledgments. We thank Walter Till, Eric Gouda, Wendy Applequist, and two anonymous reviewers for their critical comments that improved the manuscript. The curators of the following herbaria allowed the study of their collections: CHIP, CIB, CICY, CORU, ENCB, GH, HAL, HEM, IBUG, IEB, K, MEXU, MICH, MO, SERO, UAMIZ, UC, US, and XAL. Jorge Novoa-Ramos, Rodrigo Hernández-Cárdenas, and Nayeli GomezEscamilla helped us during fieldwork. We want to express our gratitude to Edith González-Rocha for helping us with the figures and to Ivonne Nayeli GomezEscamilla for drawing the distribution map.

\section{Literature Cited}

Aguirre León, E. 1986. Epífitas. Pp. 113-119 in A. Lot \& F. Chiang (editors), Manual de Herbario. Consejo Nacional de la Flora de México, Mexico City.

Brown, G. K. \& R. G. Terry. 1992. Petal appendages in Bromeliaceae. Amer. J. Bot. 79: 1051-1071.

Espejo Serna, A. 2012. El endemismo en las Liliopsida Mexicanas. Acta Bot. Mex. 100: 195-257. 


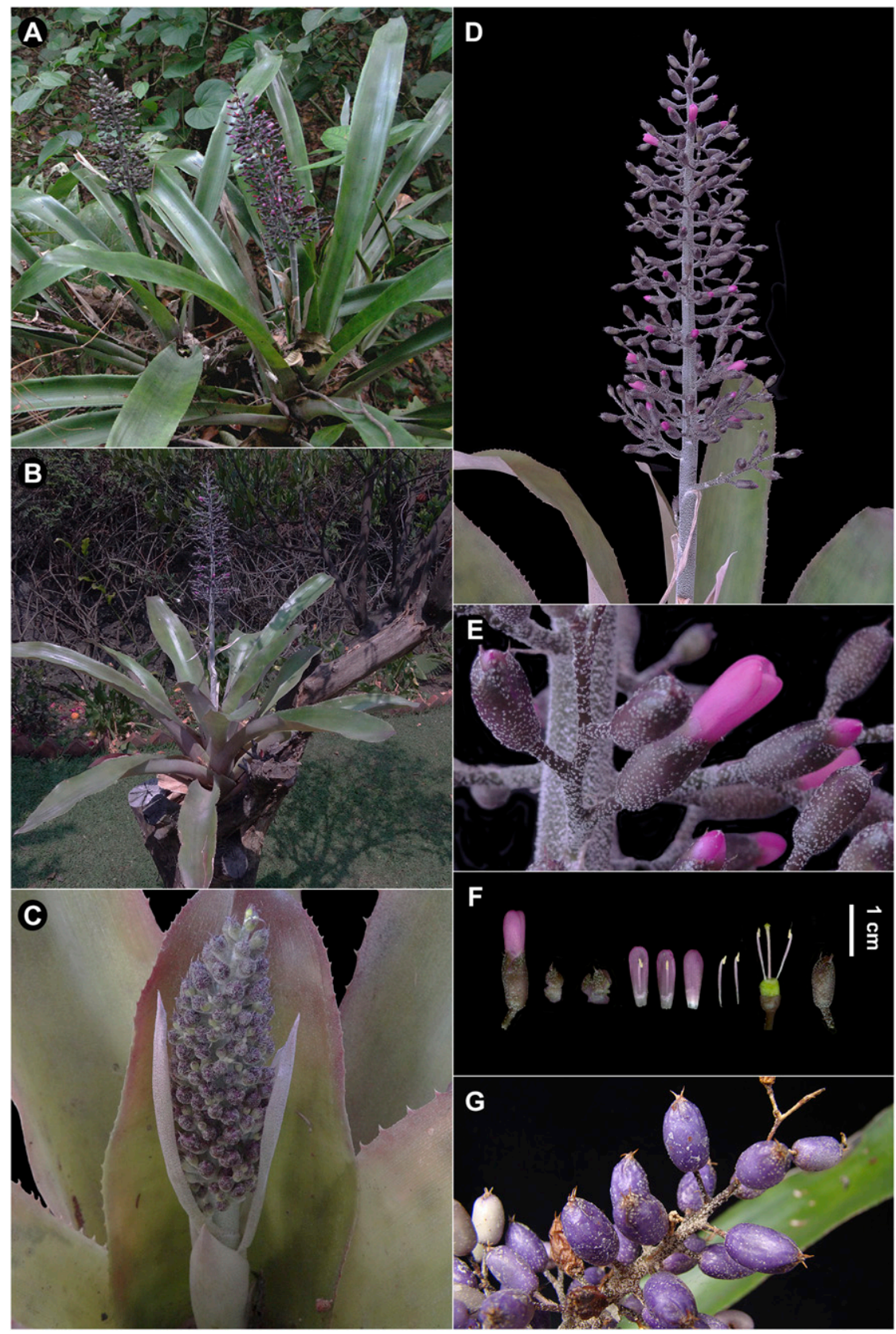

Figure 4. Aechmea lueddemanniana (K. Koch) Brongn. ex Mez. —A. Habit. -B. Plant. —C. Young inflorescence. —D. Mature inflorescence. —E. Inflorescence detail. —F. Flower dissected. —G. Fruits. Based on B. Pérez-García 1225 (UAMIZ). Photos: A, Aniceto Mendoza-Ruiz; B-G, A. Espejo-Serna.

Espejo-Serna, A. \& A. R. López-Ferrari. 1994. Bromeliaceae. Pp. 4-49 in Las Monocotiledóneas Mexicanas, una Sinopsis Florística 1. Lista de Referencia, Parte III. Bromeliaceae, Burmanniaceae, Calochortaceae y Cannaceae. Consejo Nacional de la Flora de México, Universidad Autónoma Metropolitana y Comisión Nacional para el Conocimiento y Uso de la Biodiversidad, Mexico City.
Espejo-Serna, A., A. R. López-Ferrari, I. Ramírez-Morillo, B. K. Holst, H. E. Luther \& W. Till. 2004. Checklist of Mexican Bromeliaceae with notes on species distribution and levels of endemism. Selbyana 25: 33-86.

López-Ferrari, A. R., A. Espejo-Serna, J. Ceja-Romero \& A. Mendoza-Ruiz. 2011. Aechmea aenigmatica (Bromeliaceae; 
Bromelioideae) una nueva especie del estado de Oaxaca, México. Acta Bot. Mex. 95: 1-9.

Magaña, R. P. 1986. La Familia Bromeliaceae en la Costa de Jalisco. Thesis [Tesis de licenciatura en Biología], Facultad de Ciencias, Universidad Nacional Autónoma de México, Mexico City.

McVaugh, R. 1989. Bromeliaceae. Pp. 4-79 in R. McVaugh (W. Anderson, editor), Flora Novo-Galiciana: A Descriptive Account of the Vascular Plants of Western Mexico, Vol. 15. University of Michigan Press, Ann Arbor.

Mez, C. 1896. Bromeliaceae. Pp. 1-990 in C. de Candolle (editor), Monographiae Phanerogamarum G. Masson, Paris.

Ramírez Delgadillo, R., O. Vargas P., H. J. Arreola N., M. Cedano M., R. González T., L. M. González V., M. Harker, et al. 2010. Catálogo de Plantas Vasculares de Jalisco. Universidad de Guadalajara, Sociedad Botánica de México, Universidad Autónoma Metropolitana, Guadalajara.

Ramírez-Díaz, C. J., I. Ramírez-Morillo, G. Carnevali, J. P. Pinzón, G. Zizka \& B. Holst. 2019. Notes on the Androlepis Alliance, a lineage of Bromelioideae (Bromeliaceae) with high morphological diversity. J. Bromeliad Soc. 68: 140-151.

Sass, C. \& C. Specht. 2010. Phylogenetic estimation of the core Bromelioids with an emphasis on the genus Aechmea (Bromeliaceae). Molec. Phylogen. Evol. 55: 559-571.

Scharf, U. \& E. J. Gouda. 2008. Bringing Bromeliaceae back to homeland botany. J. Bromeliad Soc. 58: 123-129.

Smith, L. B. \& R. J. Downs. 1979. Bromelioideae (Bromeliaceae). Flora Neotropica 14: 1493-2142.

Smith, L. B. \& W. J. Kress. 1989. New or restored genera of Bromeliaceae. Phytologia 66: 70-79.

Smith, L. B. \& W. J. Kress. 1990. New genera of Bromeliaceae. Phytologia 69: 271-274.

Thiers, B. $2020+$ [continuously updated]. Index Herbariorum: A global directory of public herbaria and associated staff. New York Botanical Garden's Virtual Herbarium. <http:// sweetgum.nybg.org/science/ih/>.

Vázquez-García, J. A., J. J. Reynoso D., Y. L. VargasRodríguez \& H. G. Frías U. 2000. Jalisco-Costa Norte: Patrimonio Ecológico, Cultural y Productivo de México. Libro en versión electrónica. Universidad de Guadalajara, Guadalajara.

Appendix 1. Specimens examined.

Aechmea mexicana Baker. MEXICO. Chiapas: D. E. Breedlove 29306 (ENCBx2, MO); D. E. Breedlove 33088 (MEXUx2); D. E. Breedlove 49919 (ENCB); D. E. Breedlove \& A. R. Smith 22234 (ENCBx2). Oaxaca: A. García-Mendoza et al. 2917 (MEXU); G. Juárez G. \& A. Martínez F. 840 (MEXU); G. Martínez Calderón 563 (GH); R. E. Schultes \& B. P. Reko $550 B(\mathrm{GHx} 2) ;$ R. Torres C. 12160
(MEXU); R. Torres C. \& L. Cortés A. 11457 (MEXU); R. Torres C. \& E. Martínez S. 10985 (IEB, MEXU). Puebla: F. Basurto \& $R$. Patrón 406 (MEXUx2); H. Bravo H. 552 (MEXU); A. Campos V. et al. 36 (MEXU); M. Miguel-Vazquez et al. 258 (UAMIZx4); F. Miranda 3044 (MEXUx2); P. Tenorio L. et al. 12615 (MEXU). San Luis Potosí: J. B. Alcorn 2572 (MEXU); C. R. Beutelspacher B. 33 (MEXU); C. R. Beutelspacher B. s.n. (MEXUx4); R. Merrill King 4287 (MICH, US); J. Rzedowski 10154 (ENCB, MICHx2, USx2). Veracruz: E. Bourgeau 3106 (Kx2); H. Bravo H. 224 (MEXU); H. Bravo H. 225 (MEXU); G. Castillo C. 1281 (IEB, MEXU, XAL); C. Durán E. \& S. Avendaño R. 1116 (XAL); A. Espejo et al. 7029 (UAMIZx2); I. Gómez Ponce de León \& A. Velazquez M. 20 (CORUx2, XAL); I. Gómez Ponce de León \& A. Velázquez M. 22 (CORU, XAL); I. Gómez Ponce de León \& A. Velazquez M. 25 (CORU, XAL); L. Gutiérrez C. s.n. (CORU [photo by M. Cházaro B., IBUG]); I. Kelly 224-A (US); I. K. Langman 3620 (USx2); A. Lot H. 1054 (GH, MO); V. E. Luna M. et al. 763 (MEXU, XAL); F. Miranda 4889 (MEXUx2); H. Oliva R. \& S. Ramírez C. HOR-735 (CORU); C. A. Purpus 15371 (GH); C. A. Purpus 15379 (MICH, UC); F. Ramón F. et al. FRHF 221 (CORU, XAL); R. Robles G. 451 (XAL); P. E. Valdivia Q. 1568 (CICY); F. Ventura A. 3127 (ENCB).

Aechmea lueddemanniana (K. Koch) Brongn. ex Mez. MEXICO. Chiapas: D. Álvarez \& A. Chambor 4742 (MEXU); D. E. Breedlove 15682 (MICH); J. I. Calzada et al. 9688 (MEXU, XAL); G. Carnevali et al. 4401 (CICY); G. Davidse et al. 20443 (MEXU, MO); A. Durán F. 1126 (HEM); A. Durán F. \& S. Levy T. 141 (MEXUx2); O. Farrera S. 1815 (CHIP); N. Martínez-Meléndez 1294 (HEM); E. Martínez S. 17165 (MEXU); E. Martínez S. 17612 (MEXU); E. Martínez S. 17975 (MEXUx2); E. Martínez S. M21255 (MEXU); E. Martínez S. M-21283 (MEXU); E. Martínez S. \& M. A. Soto A. 18663 (MEXU, MO); E. Martínez S. \& M. A. Soto A. 18738 (MEXU); H. E. Moore Jr. 2575 (GH, US); B. Pérez G. et al. 1225 (UAMIZ); F. Vazquez B. 927 (XAL). Oaxaca: E. Bravo \& J. Gutiérrez 207 (UAMIZ); J. Rivera H. et al. 689 (SERO); J. Rivera H. et al. 1232 (MEXU, SEROx2); R. Torres C. et al. 853 (MEXU). Veracruz: R. Acevedo R. 122 (IEB, MEXU, XAL); R. Acevedo $R$. 892 (XAL); R. Acosta P. et al. 3049 (CIB); S. Avendaño R. \& R. Sánchez 1237 (XAL); H. Bravo H. s.n. (US); C. R. Beutelspacher B. 2 (MEXU); R. Cedillo T. 3134 (MEXU, MO); Brigada Dorantes 2812 (CICY, MEXUx2, XAL); J. Dorantes S. 3230 (XALx2); A. Franco M. et al. 55 (XAL); A. Franco M. et al. 92 (XAL); G. Ibarra M. et al. 2506 (MEXU, XAL); T. Kroemer et al. 1900 (CORU); T. Kroemer et al. 1955 (MEXU, XAL); E. Lopez P. 17 (XAL); E. Lopez P. 126 (XAL); E. Lopez P. 217 (XAL); E. Matuda 38669 (MEXU); P. Maury 5597 (GH); L. I. Nevling \& A. Gómez-Pompa 2535 (MEXU); F. Ponce C. 280 (IEB, MEXUx2, MO, US);A. Rincón G. \& C. Durán E. 1485 (MEXU, XAL); A. Rincón G. et al. 2304 (XAL); J. Rivera H. et al. 1232 (MEXU, SER0x2); A. Torres S. \& A. Campos V. 244 (MEXU); P. E. Valdivia Q. 285 (XALx2); Brigada Vazquez 803 (XAL); M. Vazquez T. 803 (XAL). 